\title{
Exploration Of Surface-Programme Relationship As A Basis To Promote Hand Hygiene In Healthcare Waiting Area Setting
}

\begin{abstract}
Within a healthcare facility, surface directly correlates with the existing healthcare programme, as it allows the programme to perform in the space. In particular, this surface-programme relationship contributes towards the occurrence of disease transmission as contaminated surfaces have a significant impact on infection potential when healthcare staffs or patients come into contact with these surfaces. This paper presents a preliminary study that explores the spatial layout of a particular healthcare waiting area setting. The surface-programme relationship is analysed to reveal the potential of contact between surface and users. The results of observation and analysis indicated critical areas in the layout where hand hygiene practice becomes essential. It provides a recommendation on how hand hygiene could be promoted through the spatial configuration to prevent the possibility of infection. Overall, this paper gives a new perspective and a better understanding of how to respond to the healthcare-associated infection generally and the industrial demand on technological advancement in healthcare settings specifically.
\end{abstract}

\section{Introduction}

The implication of the relation of surface with spatial programme in healthcare facility building is argued to be even more significant compared to other building programmes since it concerns patient outcomes. A strengthened relation of surface and healthcare programme could mean a better working environment as it offers greater efficiency and productivity to which many pieces of research have shown how these offers lead to the patient outcome [1]. In particular, better thought of the performance of surface could improve the patient outcomes as it could affect the infection potential within the healthcare facility [1].

The relation between surface and disease transmission in a healthcare setting has resulted in the evidence which urges us to perform various prevention acts, including cleaning procedure [2]. Furthermore, the urgency to perform adequate cleaning has been explored as well, both clinically and in terms of infection control [2]. Cleaning, however, usually relies on the visual condition of the surfaces [3]. This implicates on how the cleaning occurs in the healthcare setting environment which consequently affecting the infection control. Relying on visual condition reduces our awareness towards the disease threat, which actually exists. Table surfaces, handrail, or door handle, which all appear 'clean' to us, could be contaminated. The as-if-it-isactually-clean appearance of these surfaces would implicate the neglect of thorough cleaning procedure. This is why hand hygiene is argued to play a significant role in the prevention of disease transmission. "Hand hygiene is accepted as the most important infection prevention measure to reduce transmission of pathogens in hospital" [4]. An adequate hand hygiene measure in healthcare settings environment could help to strengthen the overall cleaning procedure. Consequently, proper hand hygiene would reduce the up to $20 \%$ estimation of hospital-associated infection occurrence that is attributed to the environmental surfaces caused by direct transmission from touching the contaminated surfaces [5].

In retrospective, the importance of understanding surface performance on its relation with spatial programme opens an urge to further discuss hand hygiene performance in accordance with the healthcare programme. Conducting an effective hand hygiene measure within a healthcare setting environment, which corresponds to the healthcare programme, will benefit us with the higher rate of infection prevention.

\section{Hand Hygiene Measure in Healthcare Setting}

\subsection{High Touch Surfaces and Hand Hygiene}

Many studies have explored strategies to improve hand hygiene compliance, each of which is looking at various setting in a healthcare building such as ICU, NICU, and wards [6]. While there are researches on the hospitalwide setting as well, research that discusses complying hand hygiene improvement in waiting area needs further discussion. Exploring hand hygiene compliance in such common spaces in a healthcare setting is important, as we are actually unaware of infection occurrence within the medical-surgical rooms as in the common spaces of a healthcare setting such as waiting area [7].

The presence of inanimate surfaces and objects in a waiting area, which is considered to be high-touch yet uncritical is, in particular, be the reason why studying the hand hygiene compliance in waiting area is necessary. An inanimate surface is shown to have a critical role in spreading infectious pathogens, both 
directly and indirectly, once it is contaminated [8]. Assuming that the surfaces on the touched objects are contaminated, which in this paper will further be referred as fomites, thus the high frequency of touch on these objects means a higher possibility of contact transmission to occur. The transmission is likely to happen because the higher touch frequency on fomites is in the inverse with the lifespan of the pathogen to remain infectious.

In a waiting area of a healthcare setting, various inanimate yet uncritical objects could be present, such as chairs, tables, fan, board, etc. Among these objects, chair, for example, can be considered as one on which high-touch surfaces present. From here, let us take heed towards a bed-rail in a care unit that is categorised among the high-touch surface although it is not a critical object. Bed-rail, which is likely to be touched by a less number of people compared to other objects in common space where a higher number of people are present, is considered urgent enough to be included in the cleaning procedure, terminal cleaning for instance. Despite being thought as an inadequate cleaning procedure [4]. In retrospective, the handrest of a chair in a waiting area can be assumed as significant to be included in the cleaning measure as well since compared to the bed-rail, the chair-handrest in a waiting area is less involved in the overall cleaning procedure in a healthcare facility.

Moreover, "any hand-touch site that escapes cleaning offers a potential reservoir for hospital microbes" [9]. Consequently, a possible measure in the waiting area that is open to us is complying as adequate hand hygiene as possible. This way, despite the condition of the waiting area that is more likely to escapes the cleaning procedure, as there are less critical surfaces/objects within, hand hygiene would still improve the condition, hence reducing the infection potential.

\subsection{Spatial Configuration and Hand Rub Placement}

World Health Organization (WHO) suggests that one of the ways to improve hand hygiene implementation in a healthcare facility is by exploring a systemic change on the compliance itself [6]). This exploration includes the discussion on the essential aspect of the system that is "the readily-accessible alcohol-based hand rub at the point of care" [6]. This crucial aspect especially indicates the adequate placement of the hand rub as a result of positioning hand hygiene measure and the spatial healthcare programme upon the spatial configuration of the healthcare facility building.

A study conducted by [9] explores five aspects of hand hygiene measure, four of which are directly related with the spatial healthcare programme and the spatial configuration of the healthcare setting in observation. These four aspects include hand hygiene upon entering and exiting the medical room (use of alcohol rub or hand wash) and hand contact with near-patient sites such as bed frame and bedside locker and far-patient sites such as filing cabinet and keyboard [9]. Besides, WHO strongly recommends the implementation of performing hand hygiene before and after touching a patient and after touching any inanimate surfaces [6]. Simply saying, the above aspects can be categorised into two main body discussions of achieving an adequate hand rub placement, that are 1) the transition between spaces/rooms and 2) the possible contact with existing surfaces.

First, an appropriate contact precaution requires an adequate hand hygiene measure to be performed before entering any particular room in a healthcare setting [10]. Fomites touched by visitor or healthcare worker in the common area of the healthcare building such as waiting spaces could result in the transmission of the pathogen to other spaces which are specially treated and aimed to be as clean as possible; hence the cleaning procedure like terminal cleaning exist. However, the possibility of the pathogen on the fomites within particular rooms to be transmitted outside the room needs to be avoided as well so as the spread of the pathogen could be reduced.

Second, the placement of hand rub in the waiting area needs to consider where the touch is likely to occur. This is as important as the first category, arguing that placing hand rub in a location as near and visible as possible to where the fomites are presented could be a gentle 'reminder' to both visitor or healthcare worker to use the hand rub. There is an acceptance association between hand hygiene and touch transmission [4, 9] and although hand significantly contributes on the overall the reduction of the healthcare-associated infection potential, personal factors eventually affect the measure [9]. Placing the hand rub in several spots near the seating area within the waiting space, for example, might in a way overcome the personal factor in concern.

\section{Performing Surface-Programme Relation Analysis}

In order to reveal how the surface-programme relation in a healthcare facility could enhance the hand hygiene measure thus reducing the infection potential, a set of qualitative and quantitative study is conducted. The study took place in the waiting area of a healthcare facility located in a university campus environment in Depok, Indonesia. The study involves a set of contextual observation, health worker interviews, and analysis. 
The observation and interview part of the study is conducted to understand the healthcare service provided in the observed healthcare facility. In particular, it looks at the healthcare programmes which are occurred in or through the waiting space. This part of the study resulted in the overall mapping of the healthcare programme and its occurrence in the waiting space. Moreover, this part of the study is accompanied by a quantitative observation of contacts between users and the existing surfaces. Contacts occurred on sample surfaces such as doors, chairs, and table tops were counted and mapped every 10 minutes during 120 minutes. Also, all of the conducted observations were done in a condition where the observers were mingled with other users, thus reducing unwanted reactions from the users which could affect the result of the study.

Following the observation and interview, two analysis stages are conducted, each of which explores the potential contact occurrence in the observed waiting area and their relations to hand rub placement. The first analysis looked at the transition from the waiting area to other space. Meanwhile, the second stage looked at the indicative areas wherein fomites present.

\section{Exploring Surface-Programme Relationship}

Based on the observation, the healthcare programme of the facility which directly related to its waiting area is divided into three healthcare services which are lab check services, emergency services and other medical services such as general medical examination, medical rehabilitation, dentistry, and geriatric. Sequentially, the three healthcare services have similar activity programme, starting from entering the facility, which in this case directly into the waiting area, registering, checking general health condition (preliminary), entering examination room, going to the pharmacy and cashier and leaving the facility.

\subsection{Hand Rub Placement upon Leaving and Entering the Waiting Area}

In the first analysis stage, the study is looking at the relation between the programme and spatial configuration of the waiting area in regards to the possible act of both visitor or healthcare worker to either leaving or entering the waiting area. This part of analysis is conducted to examine the necessary placement of the hand rub before each subject leaves or enters the waiting area.

The healthcare programme is firstly identified for its possible leaving and entering the waiting area act. From the identification, it is shown (see Figure 1) that there are several possible leaving and entering the waiting area acts within the overall healthcare sequence. For example, one who is having a general medical examination service will have to leave the waiting area at least once that is when he/she is entering the examination room. The identification also included the move of a person from one area to another within the whole waiting space, for example, the same person who is having the medical examination service, will have to move from the waiting area in the first floor to the second floor where he/she has to do the preliminary check-up. In addition, the same person will also leave the waiting area after he/she completed the overall programme.

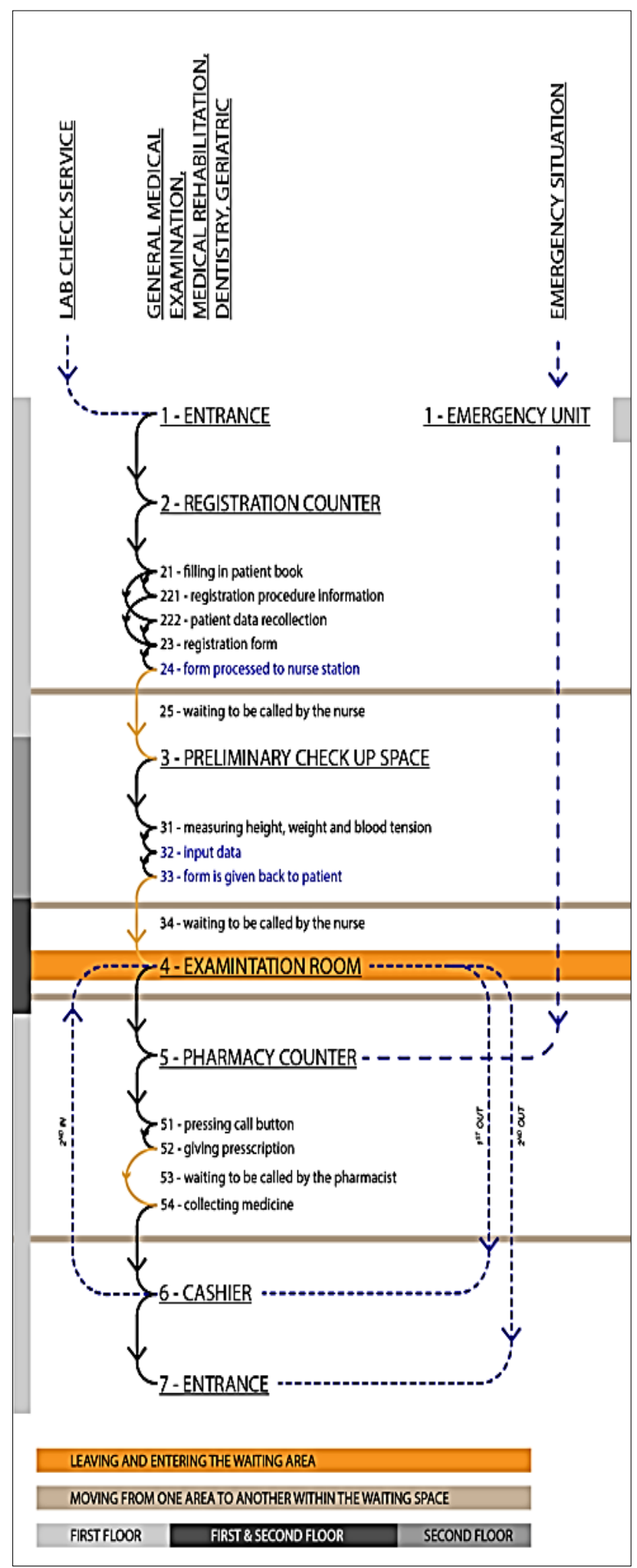

Fig. 1. The possible entering and leaving the waiting area acts as shown in the overall healthcare programme sequence 

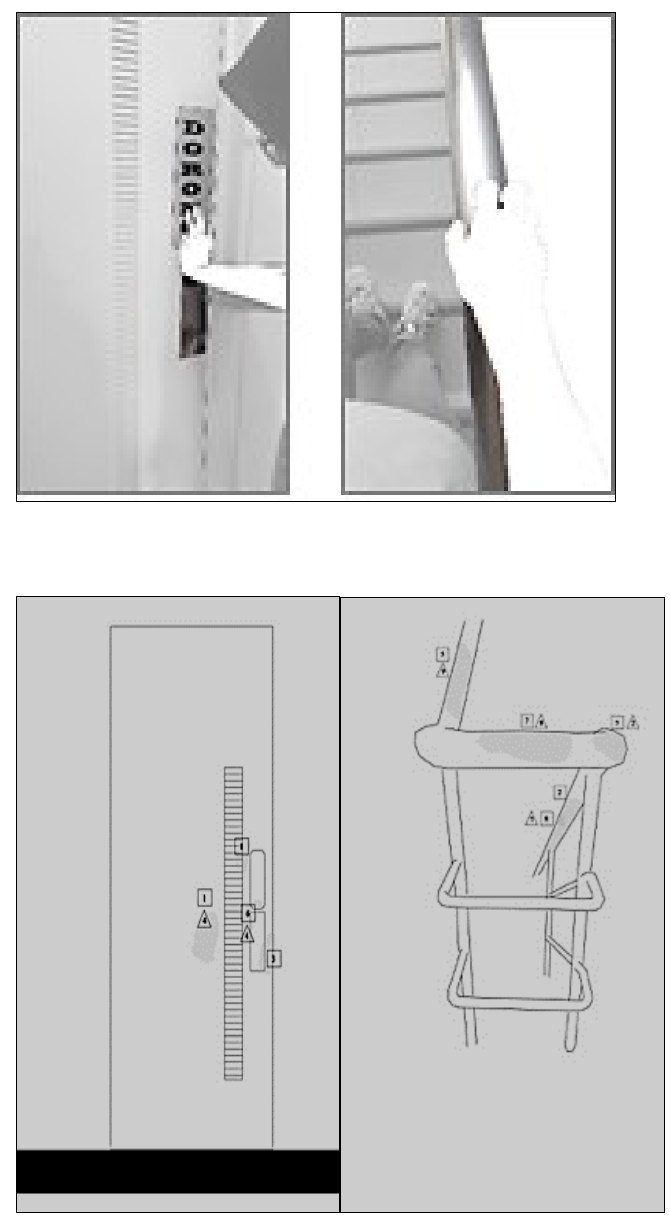

Fig. 2. Touch occurrence on the female toilet door and the stair railing surfaces (two at the top) and the counts of touch of the door and the stair railing (two at the bottom).

One of the important things to note from this part of analysis is that even the leaving and entering acts involve the act of touching certain surfaces. For example, when entering the examination room, one has to touch the door surface and even other surfaces nearby the door itself such as the wall (see Figure 2). The same person will again touch the door surface (from the examination room side this time) when he/she leaves the examination room. In particular, this needs to be considered since the door surface is more likely to be present with fomites. Door handle surface for example, which is considered as a high-touch object $[11,12]$, has more than $20 \%$ higher rate to be contaminated by infectious pathogen [12]. This implies that placing a hand rub near the door will significantly reduce the potential of touch transmission, assuming that people will use the hand rub just after they close the door behind them.

Furthermore, in the observed waiting area, the placement of hand rub needs to consider the change of area within the whole waiting space. In this study, for example, there is a change of area from one activity to another and even a change of location from one floor to another. The presence of the stair handrail, for instance, is prone to increase the touch transmission rate. This means, consideration towards the placement of such an architectural object in the process of leaving or entering one area to another within the while waiting space is necessary as well.

In addition, the quantitative observation records that the total contact number between users and both female toilet door and stair railing in the period of 120 minutes is recorded at 19 and 53 contacts respectively. However, as shown in Figure 2, the result of counting and mapping the touch indicates that the area of the touches was unevenly distributed on the surfaces.

\subsection{Hand Rub Placement Nearby High Touch Fomites}

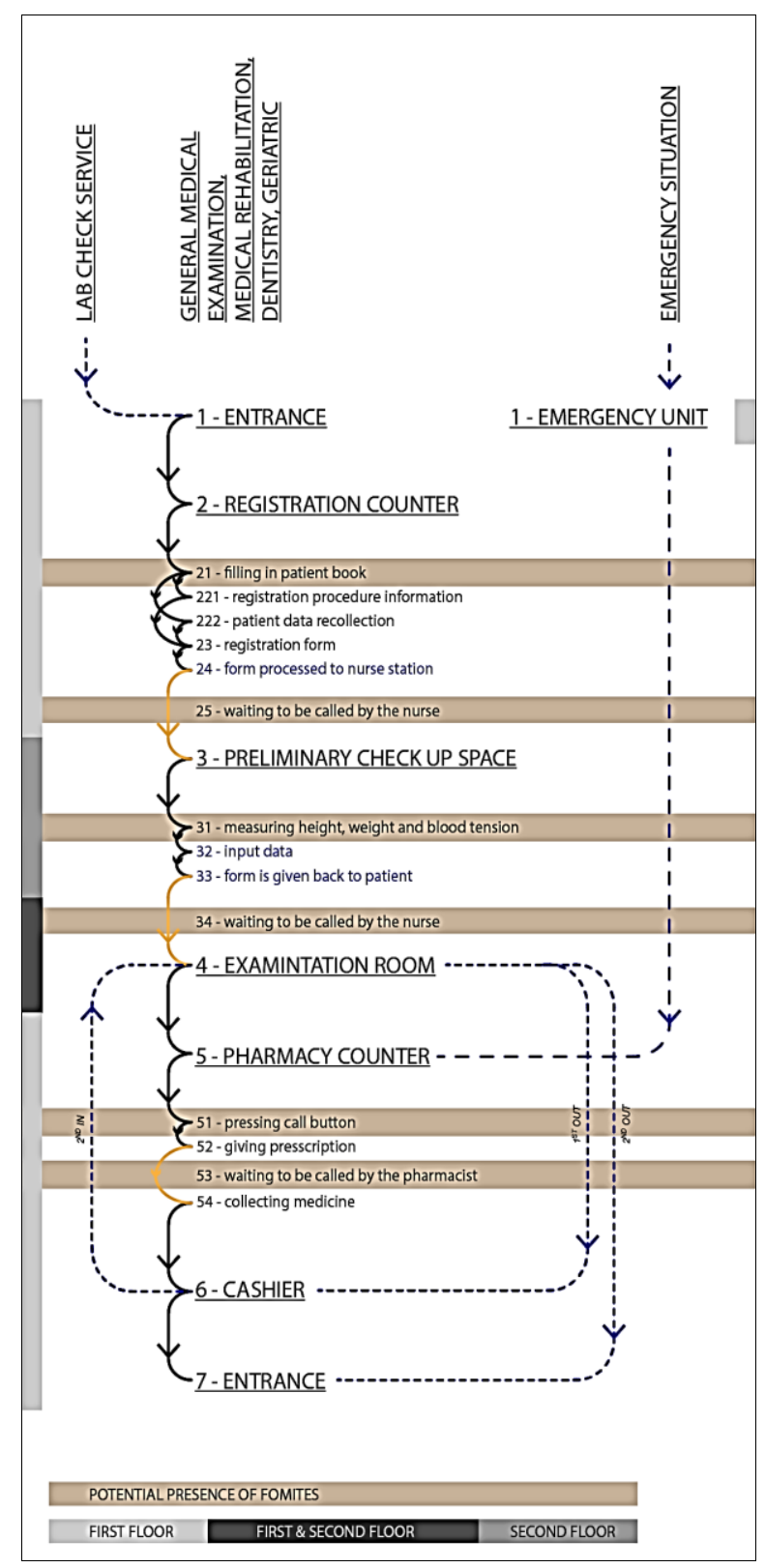

Fig. 3. The possible fomites presence in the waiting area 


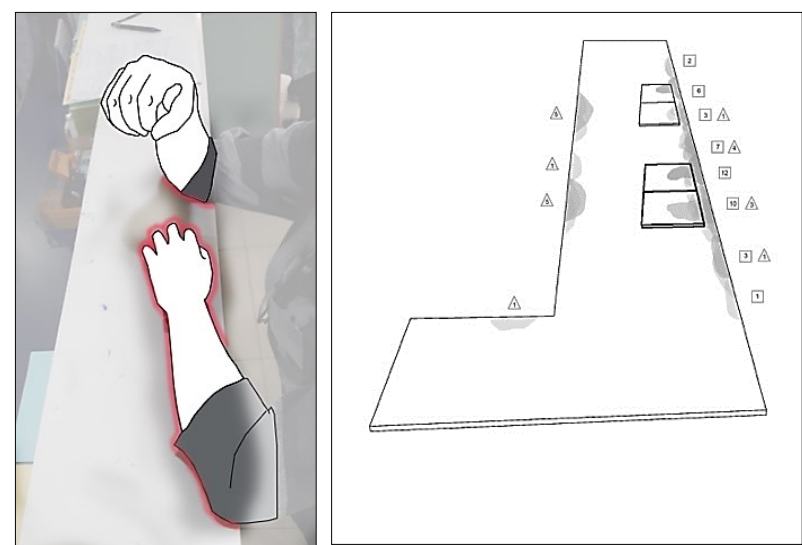

Fig. 4. Registration counter top in the waiting area presents as highly touched surface

In the second analysis stage, the result of the study covers the importance of placing the hand rub at certain spots where there is a high frequency of touching the inanimate surfaces at the waiting area. As shown in Figure 3, there are at least three different locations where the people who visited the facility were observed doing the waiting acts at different times. While waiting, those people might have touched various surfaces. For example, when they sit, it is likely that they might touch the handrest of the chair. Since many people visit the facility and use the same facility over time, therefore, it is important to place hand rub near this seating area.

Moreover, each of the activity under the healthcare programme needs to be included as well on the consideration of hand rub placement. Objects which support the healthcare activity such as registration counter (see Figure 4), should be looked into as the observation indicates that these objects are highly touched and their surfaces are prone to have fomites as well. The conducted counting of touches especially indicates this urgency. Only in the span of two hours, the registration counter top at the observed waiting area is recorded with the total of 62 touches which spread on various spots of the surface.

\section{Configuring Adequate Hand Rub Placement}

Based on the result of the two analysis stages, this study then tried to look at the existing hand rub that presents at the waiting area and proposed a suggestive recommendation of hand rub placement. Figure 6 shows the overall condition of the hand rub placement in the observed waiting area on the first floor and the potential placement spots for hand rub as well.

The mapping diagram as shown in Figure 6, indicates that the healthcare facility has provided several required hand rub spots. For example, hand rubs have been installed on the wall near the doors, which lead to the examination room. Although the installation might need to be reconfigured as the observation found an inappropriate instalment spot where the presence of some chairs blocks the hand rub. However, several spots have been identified to require hand rub placements, as there is an absence of hand rub at these required spots.

In addition, the above suggestions are based on a rather general observation of the surface-programme relationship. Thus, a further study which carefully looked at the geometrical aspect of the existing placement of hand rubs such as size and position and its visibility is necessary.

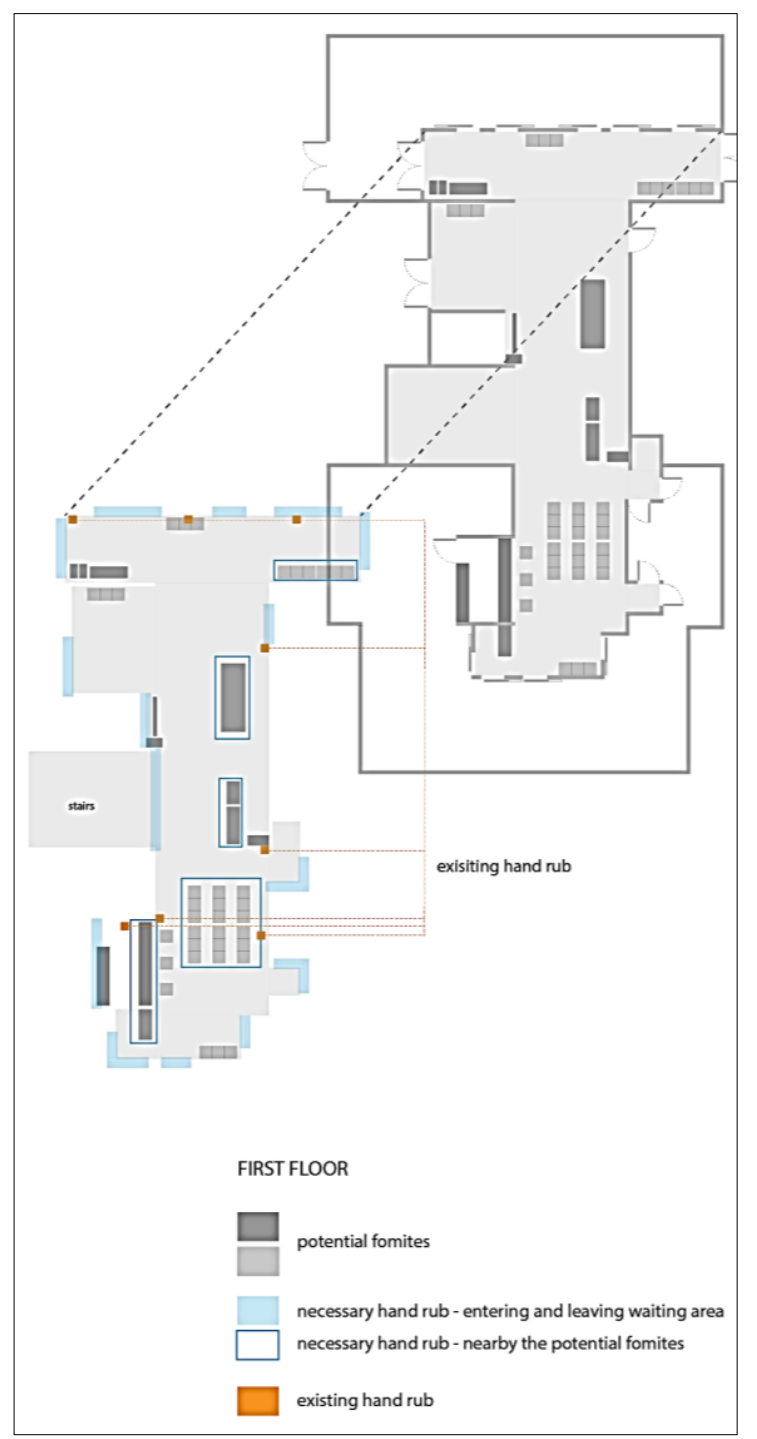

Fig. 5. Diagram of hand rub placement mapping 


\section{Conclusion}

The study reveals that the relation between surface and healthcare programme of a particular healthcare waiting area setting could be explored to find if there is an adequate configuration of hand rub in the observed waiting area. In particular, the result of the study shows that in order to promote proper hand hygiene compliance, in-depth analysis as performed within this study has the potential to reveal the required hand rub placement.

The result also implies that in the current high demand on the technological application in healthcare setting, careful and thorough consideration of placement of objects needs to be conducted first. Specifically for the focus in this study, in-depth analysis and understanding towards the adequate hand hygiene measure which includes the placement of hand rub indicate the importance to make sure that a good system change is in place. Not in technological advancement manner for effectivity and efficiency, but in the overall consideration of the infection occurrence.

The study within this paper is funded by the General Directorate of Research and Higher Education of Republic of Indonesia under the Penelitian Dasar Unggulan Perguruan Tinggi (PDUPT) 2018 Grant. The data presented in this study is collected by a team of five: Afifah Karimah, Amani Tedjowongso, Gracia C. Varani, Khalda Fadhilah A., and Vincent Khosasih.

\section{References}

1. M. Mourshed, Y. Zaho, J. Env. P. 32, 4 (2012)

2. P.C. Carling, J. Perkins, J. Ferguson, A. Thomasser, ICHE 35, 11 (2014)

3. P.A. Sharpe, M.G. Schmidt, HERD J. 5, 1 (2011)

4. C. Zimring, M.E. Denham, J.T. Jacob, D.B. Kamerow, N. Lenfestey, K.K. Hall, A. Kasali, D.Z. Cowan, J.P. Steinberg, HERD J. 7(Suppl) (2013)

5. J.P. Steinberg, M.E. Denham, C. Zimring, A. Kasali, K.K. Hall, J.T. Jacob, HERD J. 7(Suppl) (2013)

6. World Health Organization, WHO Guidelines on Hand Hygiene in Health Care: A Summary (2009)

7. D. Lee, Long-Term Living 61, 2 (2012)

8. K.A. Reynolds, P.M. Watt, S.A. Boone, C.P. Gerba, Int. J. Environ. Health Res. 15, 3 (2012)

9. S.J. Smith, V. Young, C. Robertson, S.J. Dancer, J. Hosp. Infect. 80, 3 (2012)

10. G. FitzGerald, G. Moore, A.P.R. Wilson, J. Hosp. Infect. 84, 1 (2013)

11. C.L. Murphy, D.A. Macbeth, P. Derrington, J. Gerrard, J. Faloon, K. Kenway, S. Lavender, S. Leonard, A. Orr, D. Tobin, P. Craig. Infect. Dis. \& Health. 16, 4 (2011)

12. C.C. Olise, I.A. Simon-Oke, J. Public Health Nutr. 1, 1 (2018)
(Incomplete reference. Please include title etc for a complete references). 